\title{
Weyl gravity as general relativity
}

\author{
James T. Wheeler*
}

July 9,2014

\begin{abstract}
When the full connection of Weyl conformal gravity is varied instead of just the metric, the resulting vacuum field equations reduce to the vacuum Einstein equation, up to the choice of local units, if and only if the torsion vanishes. This result differs strongly from the usual fourth-order formulation of Weyl gravity.
\end{abstract}

\section{Introduction}

General relativity accounts in exquisite detail for nearly all gravitational phenomena. Thorough tests of its predictions have met with repeated success, as alternative theories have required modification or abandonment. Still, some alternative theories survive and provide, at the very least, further tests of our understanding. From the introduction of the equivalence principle in 1908, through the presentation of the final field equations in 1915, development of general relativity was rapid [1], yet no faster than the introduction of a second relativistic theory of gravity by Weyl, Bach and others in the years from 1918 to 1924 . This second theory, called conformal gravity or Weyl gravity, remains a topic of active discussion despite its higher order field equations. We hope to clarify the reason why it has been difficult to distinguish these first two relativistic gravity theories. Specifically, we show that the when all of the connection fields of conformal gravity are varied independently instead of the usual fourth-order, metric-only variation, the torsion-free solutions of the two theories differ only in that the field equations of conformal gravity are unchanged by the use of arbitrary local choices of units.

Beginning in 1918, Weyl [2], 3] and Bach [4] developed a theory of gravity based on the conformally invariant Weyl action

$$
S=\alpha \int C_{a b c d} C^{a b c d} \sqrt{-g} d^{4} x
$$

Bach adds to this a second term quadratic in the dilatational curvature. Variation leads to the Bach equation,

$$
D_{\alpha} D_{\beta} C^{\mu \alpha \nu \beta}-\frac{1}{2} C^{\mu \alpha \nu \beta} R_{\alpha \beta}=0
$$

An alternate approach [4 makes use of the Gauss-Bonnet integral for the Euler character [5] to write the equivalent action, $S^{\prime}=2 \alpha \int\left(R_{a b} R^{a b}-\frac{1}{3} R^{2}\right) \sqrt{-g} d^{4} x$, and field equations depending only on the Ricci tensor and its derivatives.

From the point of view of quantum theory, Weyl conformal gravity has an important advantage and an equally important disadvantage. Power counting suggests that the curvature-quadratic action, eq.(1), is renormalizable. However, the presence of fourth order derivatives in the field equations, eq.(2), is generally associated with non-unitarity. Rather than entering into the controversy surrounding these observations (see, e.g., [6], [7]), we propose a full connection variation of eq.(11). We show that torsion-free solutions to the

\footnotetext{
*Utah State University Department of Physics 4415 Old Main Hill, Logan, UT 84322-4415; jim.wheeler@usu.edu
} 
resulting field equations lead purely to the second order field equation of general relativity, modified to have local dilatational covariance. Within this alternative approach, the debate over unitarity becomes moot.

Discussion also surrounds certain solutions to the Bach equation. Bach's generalization of the Schwarzschild solution [4, for example, has been developed into a model to explain galactic rotation curves [8], but may fail at solar system scales [9], [10]. This discussion has faded in importance as many more independent consequences and tests of dark matter have emerged. Again, within our current presentation, these considerations do not apply.

It is interesting to speculate that conformal gravity with full connection variation, having a dimensionless action, might give rise to a renormalizable quantization of general relativity or contribute to a deeper understanding of the relevance of twistor string theory [11].

Solutions for the metric in conformal gravity are determined only up to an overall multiple, forming elements of conformal equivalence classes, $g_{\alpha \beta} \in\left\{e^{2 \varphi} g_{\alpha \beta} \mid\right.$ all $\left.\varphi\left(x^{\mu}\right)\right\}$. As long as the dilatational potential, the Weyl vector, is a pure gradient, it is consistent to regard this factor as a choice of local units. Given this requirement for conformal equivalence classes of solutions, it becomes necessary to ask when a given metric is conformal to a metric satisfying the Einstein equation. This question was first addressed in 1924 by Brinkmann [12, who found a set of necessary and sufficient conditions for a space to satisfy the vacuum Einstein equation up to a conformal transformation. These expressions have the disadvantage of depending on the conformal transformation itself, so that one simultaneously checks for the existence of a suitable transformation and finds it. In 1963, Szekeres [13] used spinor techniques to separate the existence of a conformal transformation to an Einstein space from the problem of finding that transformation. As expected from the fact that a conformal transformation changes the Ricci tensor by terms involving the second derivative of the conformal factor, there are two integrability conditions. Subsequent work refines or gives different expression to these results [14].

It is crucial to the present investigation that the Bach equation (for which torsion is always assumed to vanish) has solutions which are not solutions to the vacuum Einstein equation. The need for equivalence classes of metrics complicates this. It was not until 1984 that Schmidt [15] showed conclusively the existence of solutions to conformal gravity which are not conformally equivalent to Einstein spaces (i.e., spaces for which the Ricci tensor equals a constant times the metric). Subsequently, additional non-Einstein space solutions were found by Nurowski and Plebanski [16], and six more solutions by Liu, Lü, Pope and VázquezPoritz [17. The existence of non-Einstein solutions to fourth-order conformal gravity demonstrates that the stronger restrictions that we describe here are not vacuous - our results below demonstrate a distinct interpretation of Weyl gravity from the fourth-order theory. Since our method is natural within the context of conformal gauge theory, we will refer to conventional conformal gravity as the fourth-order theory and the method we employ as auxiliary conformal gauge theory. The name stems from the way the special conformal gauge fields act as auxiliary fields that turn the full curvature into the Weyl curvature.

Conformal gauge theory was first written down in the mid-1970s. Leading up to a conformal supergravity model, Crispim-Romao, Ferber and Freund [18] performed the first gauging of the conformal group, $O(4,2)$, writing the Weyl action in terms of the conformal curvatures. Kaku, Townsend and van Nieuwenhuizen [19] developed a similar gauging. Both this group [20, and Crispim-Romao [21] went on to write superconformal gravity theories (for a review of superconformal gravity, see [22]), and both show that the gauge field of the special conformal transformations is the Schouten tensor (equivalent to the Ricci tensor) hence auxiliary (see also [23]). Within a few years, Ivanov and Niederle [24, 25] gave a more systematic treatment of gauge theories of gravity, using techniques developed by Ne'eman and Regge [26] based on the work of Cartan [27]. Their work identifies two distinct conformal gaugings, now called the auxiliary and biconformal [28, 29] gaugings.

We use these techniques in our formulation since they have the advantage of treating each independent gauge field on an equal footing. This makes variation of all 15 gauge vectors natural, giving additional field equations beyond the Bach equation. Half the equations are easily solved, establishing the auxiliary field and showing the equivalence to Weyl gravity. These results are well-known. However, the variation of the spin connection provides another field equation, the vanishing divergence of the Weyl curvature. Our central result is to show that this equation is an integrability condition which reduces the theory to scale-invariant 
general relativity. With this change from varying only the metric to varying all of the connection fields, Weyl gravity changes from a fourth order theory into a theory of conformal equivalence classes of solutions to ordinary general relativity.

In the next Section, we develop auxiliary conformal gravity. Though our action is not initially invariant under the full conformal group, it is well-known that the field equation of the special conformal transformation gauge field reduces the action to eq.(11). From the scale-invariant action, we could perform either the fourthorder metric variation by assuming the metric form of the connection, or the gauge theory approach in which each connection component is independently varied. Writing the field equations from the latter, we show that any torsion-free solution of the new field equations solves the Bach equation. Finally, we show that the new field equation is the integrability condition that forces solutions to be conformal equivalence classes of solutions to the vacuum Einstein equation.

\section{Auxiliary conformal gauge theory}

We briefly outline auxiliary conformal gauge theory, culminating in the action and field equations. The basic construction uses group quotients to construct a fiber bundle with chosen symmetry, then modifies the base manifold and connection to give curvature ([24]-[27]). The advantage of the approach is that it keeps the curvatures and action expressed in terms of the gauge fields, making the variation straightforward. In the next Section we consider solutions.

The conformal group of spacetime has fifteen generators: six for Lorentz transformations, four translations, four special conformal transformations, and one dilatation. For each of these we have a corresponding dual 1-form: $\boldsymbol{\omega}^{A} \in\left\{\boldsymbol{\omega}_{b}^{a}, \mathbf{e}^{a}, \mathbf{f}_{a}, \boldsymbol{\omega}\right\}$ called the spin connection, the solder form, the gauge field of special conformal transformations and the Weyl vector, respectively. These, together with the group structure constants, are substituted into the Maurer-Cartan equation.

To recover Weyl gravity, we take the quotient of the conformal group by the inhomogeneous Weyl subgroup, IW , generated by Lorentz transformations, special conformal transformations, and dilatations. This quotient is a homogeneous, 4 -dim manifold, and the 1 -forms above provide its connection. Next, we modify this structure by generalizing the manifold, and by changing the connection. Changing the manifold has no effect on the local structure, but changing the connection modifies the Maurer-Cartan equation, resulting in the addition of curvature 2-forms, $\boldsymbol{\Omega}^{A} \in\left\{\boldsymbol{\Omega}_{b}^{a}, \mathbf{T}^{a}, \mathbf{S}_{a}, \boldsymbol{\Omega}\right\}$. We place two restrictions on these curvatures. First, we require the curvatures to characterize the manifold only. In general, an integral of the connection along a curve in the full space gives a conformal transformation, with integrals around closed loops equivalent to surface integrals of the curvatures. We require horizontlity: these closed loop integrals must be independent of the $\mathcal{I} \mathcal{W}$ subgroup transformations, which occurs if and only if the curvatures may be expanded in terms of the solder forms, $\boldsymbol{\Omega}^{A}=\frac{1}{2} \Omega_{c d}^{A} \mathbf{e}^{c} \wedge \mathbf{e}^{d}$, and not all fifteen connection forms. Second, we require integrability of the Cartan equations (i.e., these modified Maurer-Cartan equations). This leads to Bianchi identities for the curvatures. The Cartan equations and Bianchi identities are given in Appendix A.

The quotient construction describes only the geometry, leading us to the form for the curvatures in terms of the gauge fields, which agree with those found in [20, [21], and [24], and proivding the Bianchi identities. The physical content arises solely from the field equations, found by writing an action functional defined on the local $\mathcal{I} \mathcal{W}$-invariant principal bundle. The action is constructible from the available tensors, $\mathbf{e}^{c}, \boldsymbol{\Omega}_{B}^{A}$, together with the invariant metric and Levi-Civita tensors, $\eta_{a b}, \varepsilon_{a b c d}$. Scale invariance requires curvaturequadratic terms, and the most general even parity, $\mathcal{I} \mathcal{W}$-invariant possibility is uniquely determined (up to an overall multiple) to be

$$
\begin{aligned}
S_{\text {auxiliary }}^{\mathcal{I \mathcal { W }}} & =\alpha \int \boldsymbol{\Omega}_{B}^{A} \wedge^{*} \boldsymbol{\Omega}_{A}^{B} \\
& =\alpha \int\left(\boldsymbol{\Omega}^{a}{ }_{b} \wedge^{*} \boldsymbol{\Omega}^{b}{ }_{a}+4 \mathbf{T}^{c} \wedge^{*} \mathbf{S}_{c}+2 \boldsymbol{\Omega} \wedge^{*} \boldsymbol{\Omega}\right)
\end{aligned}
$$

where $\boldsymbol{\Omega}_{B}^{A}$ is the full $S O(4,2)$ curvature 2 -form. This does not lead to Weyl gravity, as will be shown elsewhere, but instead to a Weyl-Cartan geometry (i.e., one having nontrivial dilatation and torsion). To 
achieve Weyl gravity on the $\mathcal{I} \mathcal{W}$ bundle, we need to break the special conformal symmetry with our choice of the action. Since the curvature has already broken the translational symmetry, we expect both nondynamical torsion and non-dynamical special conformal curvature. Dropping the center term in $S_{\text {auxiliary }}^{\mathcal{I} \mathcal{W}}$, we have the $\mathcal{W}$-invariant Weyl-Bach action,

$$
S_{\text {auxiliary }}^{\mathcal{W}}=\int\left(\alpha \boldsymbol{\Omega}_{b}^{a} \wedge^{*} \boldsymbol{\Omega}_{a}^{b}+\beta \boldsymbol{\Omega} \wedge^{*} \boldsymbol{\Omega}\right)
$$

The equivalence between the first term and the original conformal gravity action is established in 20 and 24], while the vanishing of the second term is shown below. Bach's original action included both terms but with the critical value of $\beta=2 \alpha$ (in our notation). A detailed discussion of these symmetries is provided in Appendix B.

Varying the entire Cartan connection gives the field equations. This is where the difference between our approach and the usual approach to Weyl gravity occurs. In order to display the torsion dependence of the field equations explicitly, we write the field equations in a coordinate basis.

$$
\begin{aligned}
& D_{\tau}{\Omega^{\mu}{ }_{\nu} \lambda \tau}+\Omega^{\mu \lambda}{ }_{\nu}^{\lambda \alpha} T_{\alpha \tau}^{\tau}+\frac{1}{2} \Omega^{\mu \alpha}{ }_{\nu}{ }^{\alpha} T_{\alpha \tau}^{\lambda}=0 \\
& D_{\nu} \Omega^{\mu \nu}+\Omega^{\mu \alpha} T_{\alpha \nu}^{\nu}+\frac{1}{2} \Omega^{\alpha \nu} T_{\alpha \nu}^{\mu}=0 \\
& 2 \alpha{\Omega^{\mu}}_{\alpha \mu \beta}+\beta \Omega_{\alpha \beta}=0 \\
& 2 \alpha f_{\mu \nu} \Omega^{\mu \alpha \nu \beta}+\beta f_{\mu}^{\alpha} \Omega^{\beta \mu}=-\alpha \Theta^{\alpha \beta}-\beta Q^{\alpha \beta}
\end{aligned}
$$

where eq.(44) arises from the variation of $\boldsymbol{\omega}_{b}^{a}$, eq.(15) from $\boldsymbol{\omega}$, eq.(6) from $\mathbf{f}_{a}$ and eq.(17) from $\mathbf{e}^{a}$. All occurences of the torsion arise from derivatives of the solder form. The sources for the solder form equation are given by,

$$
\begin{aligned}
\Theta^{\alpha \beta} & \equiv-\Omega^{\mu \nu \rho \alpha} \Omega_{\mu \nu \rho}^{\beta}+\frac{1}{4} \Omega^{\mu \nu \rho \sigma} \Omega_{\mu \nu \rho \sigma} g^{\alpha \beta} \\
Q^{\alpha \beta} & \equiv \Omega^{\mu \alpha} \Omega_{\mu}^{\beta}-\frac{1}{4} \Omega^{\mu \nu} \Omega_{\mu \nu} g^{\alpha \beta}
\end{aligned}
$$

These sources arise because the Hodge dual is a nonlinear function of the solder form. The covariant derivatives are taken using the torsionful, Weyl-covariant, metric compatible connection

$$
\tilde{\Gamma}_{\mu \nu}^{\beta} \equiv \Gamma_{\mu \nu}^{\beta}-\left(\delta_{\mu}^{\beta} W_{\nu}+\delta_{\nu}^{\beta} W_{\mu}-g^{\alpha \beta} g_{\nu \mu} W_{\alpha}\right)+\frac{1}{2}\left(T_{\mu \nu}^{\beta}+T_{\nu \mu}^{\beta}-T_{\mu \nu}^{\beta}\right)
$$

where $\Gamma_{\mu \nu}^{\beta}$ is the usual Christoffel connection. The derivation of this and other useful relations is described in Appendix C.

Having expressed the field equations in terms of covariant derivatives satisfying $D_{\alpha} e_{\beta}{ }^{a}=0$, we may freely interchange between coordinate (Greek) and orthonormal (Latin) indices.

\section{Solving the field equations}

The system to be solved now consists of eqs.(4.9) with the form of the curvatures dictated by the conformal group. Our central result is to show that the solution is scale-invariant general relativity if and only if the torsion vanishes. Of course, if the torsion does not vanish we do not have general relativity. To complete the result, we must show that when we set the torsion to zero, $T_{\alpha \beta}^{\mu}=0$, eqs.(44]7) describe dilatationally covariant general relativity.

First, we show that the dilatational curvature, $\Omega_{a b}$, generically vanishes. We simplify the special conformal field equation, eq.(6) using the torsion-free Bianchi identity (see Appendix A) of the solder form, $\Omega_{[b c d]}^{a}=$ 
$\delta_{[b}^{a} \Omega_{c d]}$. Combining its trace, $\Omega_{b a c}^{a}-\Omega_{c a b}^{a}=-2 \Omega_{b c}$, with the antisymmetric part of field equation, eq.(6), leads to

$$
(2 \alpha-\beta) \Omega_{a b}=0
$$

Unless the arbitrary constants in the action are related by $\beta=2 \alpha$, the dilatation vanishes $\Omega_{a b}=0$ and there is no physically measurable size change. Eq.(5) is now satisfied.

Next, we find the special conformal gauge field, $f_{a b}$. With vanishing dilatational curvature, eq.(6) reduces to $\Omega_{a b c}^{b}=0$. Defining the Riemann curvature of the spin connection

$$
\mathbf{R}_{b}^{a} \equiv \mathbf{d} \boldsymbol{\omega}_{b}^{a}-\boldsymbol{\omega}_{b}^{c} \wedge \boldsymbol{\omega}_{c}^{a},
$$

the Lorentz curvature becomes $\Omega_{b c d}^{a}=R_{b c d}^{a}+\delta_{c}^{a} f_{b d}-\delta_{d}^{a} f_{b c}+f_{c}^{a} \eta_{b d}-f_{d}^{a} \eta_{b c}$. Substituting into the field equation, we readily solve,

$$
f_{a b}=-\frac{1}{2}\left(R_{b d}-\frac{1}{6} R \eta_{a b}\right) \equiv-\mathcal{R}_{a b}
$$

where the Schouten tensor $\mathcal{R}_{a b}$ may be used interchangeably with the Ricci tensor, since $R_{a b}=2 \mathcal{R}_{a b}+\eta_{a b} \mathcal{R}$. This result is well-known (20, [21, 23]) and it eliminates $f_{a b}$ from the problem. Substituting into the Lorentz curvature yields the Weyl curvature, $\Omega_{b c d}^{a}=C_{b c d}^{a}$, so the "auxiliary" field, $f_{a b}$, systematically enforces the conformal structure. Also, substituting $f_{a b}=-\mathcal{R}_{a b}=f_{(a b)}$ into the expression for the dilatation in terms of the connection, $\Omega_{a b}=f_{[a b]}+\omega_{[a ; b]}$ shows that the Weyl vector $\omega_{a}$ is a pure gradient.

These considerations show the equivalence of the auxiliary and Weyl actions, so the Bach equation must be satisfied. To see how, first observe that $\Theta_{a b}$, eq.(8), becomes the energy momentum tensor of the Weyl curvature and therefore vanishes identically in 4-dim. This identity, $\Theta_{a b}=0$ was first shown by Lanczos 30] (but see also [31, [32]). Vanishing dilatation gives $Q^{\alpha \beta}=0$ in eq.(91), reducing the right side of eq.(7) to zero. Finally, replacing $\Omega^{a b c d}=C^{a b c d}$ and $f_{a b}=-\mathcal{R}_{a b}$, in eq.(7) gives $R_{c d} C^{c a d b}=0$, which, combined with the covariant divergence of eq.(4), reproduces the Bach equation.

We conclude that all solutions to torsion-free auxiliary conformal gauge theory are also solutions to fourth-order field Weyl gravity. The converse, however, is not true. We now show that the class of solutions is equal to the set of conformal equivalence classes of Ricci-flat spacetimes.

The calculation centers on the vanishing divergence of the Weyl curvature, $D_{a} C^{a b c d}=0$, the torsion-free version of eq.(44). Clearly, this field equation distinguishes the two approaches to Weyl gravity. Without this, we would only have vanishing dilatation, a gauge with vanishing Weyl vector, and $f_{\mu \nu}=-\mathcal{R}_{\mu \nu}$ turning the curvature into the Weyl curvature, all of which reduces the problem to the usual Weyl curvature squared action together with the metric variation that gives the Bach equation. The remarkable thing is that the vanishing divergence is an integrability condition that reduces the fourth order theory to a second order theory. To see this, we choose the conformal gauge so that the Weyl vector vanishes. This makes the geometry appear Riemannian. The field equations reduce to eq.(4) and, from eq.(7), the condition $R_{a b} C^{a c b d}=0$. This latter expresses the vanishing energy-momentum of the Schouten tensor 32.

Expanding the second Bianchi identity, $R_{b[c d ; e]}^{a}=0$ in terms of the Weyl and Schouten parts then taking a trace relates the divergence of the Weyl curvature to the Schouten tensor. Using the identity $\mathcal{R}_{; d}=\mathcal{R}_{d ; a}^{a}$, from a second trace, the Bianchi identity becomes

$$
C_{b c d ; a}^{a}+(n-3)\left(\mathcal{R}_{b c ; d}-\mathcal{R}_{b d ; c}\right)=0
$$

(in $n$-dim) so the field equation may be written as

$$
\mathcal{R}_{b[c ; d]}=0
$$

This is not the well-known integrability condition,

$$
\mathcal{R}_{b c ; d}-\mathcal{R}_{b d ; c}+\varphi_{a} C_{b c d}^{a}=0,
$$


for the existence of a gauge in which the vacuum Einstein equation holds ([12], [13]). The problem is that we are in the wrong basis to see the integrability condition. Staying in the Riemannian gauge, we define a new basis,

$$
\tilde{\mathbf{e}}^{a}=e^{\chi} \mathbf{e}^{a}
$$

and require the same relations between $\tilde{\mathbf{e}}^{a}, \tilde{\boldsymbol{\omega}}_{b}^{a}$ and $\tilde{\mathbf{R}}_{b}^{a}$ as hold between $\mathbf{e}^{a}, \boldsymbol{\omega}_{b}^{a}$ and $\mathbf{R}_{b}^{a}$. The Bianchi identity remains the same, $\tilde{C}_{b c d ; a}^{a}+(n-3)\left(\tilde{\mathcal{R}}_{b c ; d}-\tilde{\mathcal{R}}_{b d ; c}\right)=0$ but the field equation differs. With the new connection given by

$$
\tilde{\boldsymbol{\omega}}_{b}^{a}=\boldsymbol{\omega}_{b}^{a}+2 \Delta_{d b}^{a c} \chi_{c} \mathbf{e}^{d},
$$

we find the well-known change in the Schouten tensor [33], with the Weyl curvature unchanged. The divergence of the Weyl curvature, however, is related to the old by $D_{a}^{(\omega)} C^{a}{ }_{b c d}=e^{2 \chi}\left(\tilde{D}_{a} \tilde{C}_{b c d}^{a}-(n-3) \chi_{e} \tilde{C}_{b c d}^{e}\right)=$ 0 . Combining this with the Bianchi identity, the field equation is now

$$
\tilde{\mathcal{R}}_{b c ; d}-\tilde{\mathcal{R}}_{b d ; c}+\chi_{e} \tilde{C}^{e}{ }_{b c d}=0
$$

and this is the integrability condition. Therefore, there exists a gauge, $\chi$, which takes $\tilde{\mathbf{e}}^{a}$ to a Ricci-flat basis.

Some care is now required. The integrability condition, eq.(11), tells us that $\tilde{\mathbf{e}}^{a}$ is conformal to a basis in which the spacetime has vanishing Ricci tensor. Let this basis be $\hat{\mathbf{e}}^{a}=e^{\xi} \tilde{\mathbf{e}}^{a}$ for some function $\xi$. Then, since $\tilde{\mathbf{e}}^{a}=e^{\chi} \mathbf{e}^{a}$, we have $\hat{\mathbf{e}}^{a}=e^{\xi+\chi} \mathbf{e}^{a}$. This means that the original basis is conformal to a Ricci-flat basis, and therefore the integrability condition must hold there as well,

$$
\mathcal{R}_{b c ; d}-\mathcal{R}_{b d ; c}+\zeta_{e} C^{e}{ }_{b c d}=0
$$

where $\zeta=\xi+\chi$. However, since we know that $\mathcal{R}_{b c ; d}-\mathcal{R}_{b d ; c}=0$ by the field equation we must also have

$$
\zeta_{e} C^{e}{ }_{b c d}=0
$$

in the original basis. For spacetimes other than Petrov types $\mathrm{O}$ or $\mathrm{N}$, this only happens if

$$
\zeta_{a} \equiv e_{a}{ }^{\mu} \partial_{\mu} \zeta=0
$$

so $\zeta=\zeta_{0}$ is at most a constant and $\hat{\mathbf{e}}^{a}=e^{\zeta_{0}} \mathbf{e}^{a}$. A constant multiplying the basis preserves the vanishing Ricci tensor, so the Ricci tensor vanishes in the original basis. While Petrov type $\mathrm{O}$ and $\mathrm{N}$ spaces are conformally Ricci flat, the Ricci tensor may not vanish in Riemannian gauge. It is worth noting that a number of the non-Einstein solutions in [17] are wavelike solutions of type N. These special cases warrant further study; type $N$ spaces are the same ones Szekeres found to be exceptional [13].

We see that the Riemannian gauge is doubly special (except possibly in type $\mathrm{O}$ or $\mathrm{N}$ spaces): both the Weyl vector and the Ricci tensor vanish in that gauge. This is part of the reason the integrability was not seen earlier. There is another reason as well, stemming from the fact that the special form $\mathcal{R}_{b c ; d}-\mathcal{R}_{b d ; c}=0$ admits Einstein spaces as solutions. We now clarify this issue.

Eq.(10) is solved by any Einstein space, $\mathcal{R}_{a b}=\frac{1}{6} \Lambda \eta_{a b}$ for constant $\Lambda$. This seems to contradict our result above. The problem is resolved if we recall that solutions are conformal equivalence classes of metrics, $\left\{e^{2 \varphi} g_{\alpha \beta} \mid\right.$ all $\left.\varphi\right\}$. Above, we showed that metrics conformal to Ricci-flat metrics comprise such a class. If we compute the condition for a space to be conformal to an Einstein space, however, eq.(11) gains a new term,

$$
\mathcal{R}_{a[b ; c]}+\chi_{d} C_{a b c}^{d}+\frac{1}{3} \Lambda \eta_{a[b} \chi_{c]}=0
$$

Since the field equation requires $\mathcal{R}_{a[b ; c]}+\chi_{d} C_{a b c}^{d}=0$, a conformal equivalence class with cosmological constant also requires

$$
\frac{1}{3} \Lambda \eta_{a[b} \chi_{c]}=0
$$


Since $\chi$ is arbitrary, the cosmological constant must vanish, $\Lambda=0$. We conclude that, while all Einstein spaces satisfy eq.(10), the only conformal equivalence class of Einstein spaces satisfying eq.(10) when expressed in the Riemannian gauge is the class with $\Lambda=0$, and therefore the Ricci-flat equivalence class.

Finally, we return to the field equations in an arbitrary gauge, so the Weyl vector is no longer zero. The Schouten tensor becomes

$$
\mathcal{R}_{a b}=\mathcal{R}_{a b}^{(\alpha)}-\omega_{(a ; b)}-\omega_{a} \omega_{b}+\frac{1}{2} \omega^{2} \eta_{a b}
$$

where $\mathcal{R}_{a b}^{(\alpha)}$ is the Schouten tensor in Riemannian gauge. But $\mathcal{R}_{a b}$ is covariant under conformal transformations, with $\mathcal{R}_{a b} \rightarrow e^{-2 \chi} \mathcal{R}_{a b}$. We may therefore evaluate it in any convenient gauge and immediately know it in any other. Now we see the importance of generically having both vanishing Weyl vector and vanishing Ricci tensor in the Riemannian gauge - evaluating eq.(13) in the Riemannian gauge now shows that

$$
\mathcal{R}_{a b}=0
$$

in every gauge.

In conclusion, we have shown that when all connection fields of conformal gravity are varied independently, solutions are conformal equivalence classes of solutions to the vacuum Einstein equation. Quantization of conformal gravity may therefore be renormalizable, ghost free, and essentially equivalent to general relativity. Investigations along these lines are ongoing. In Petrov type $\mathrm{O}$ or $\mathrm{N}$ spaces, the Weyl vector and Ricci tensor may vanish in different gauges; the dilatational curvature may not vanish when the original gauge theory action has $\beta=2 \alpha$.

\section{Acknowledgements}

The author thanks J. Trujillo, J. Hazboun, and C. Torre for illuminating discussions, an anonymous referee for urging clarification of the role of torsion, and J. Trujillo for his careful checking of many of the calculations.

\section{Appendix A: Structure equations and Bianchi identities}

The curvature 2 -forms are given in terms of the connection by of the Cartan equations for the conformal group:

$$
\begin{aligned}
\boldsymbol{\Omega}_{b}^{a} & =\mathbf{d} \boldsymbol{\omega}_{b}^{a}-\boldsymbol{\omega}_{b}^{c} \wedge \boldsymbol{\omega}_{c}^{a}-2 \Delta_{d b}^{a c} \mathbf{f}_{c} \wedge \mathbf{e}^{d} \\
\mathbf{T}^{a} & =\mathbf{d} \mathbf{e}^{a}-\mathbf{e}^{b} \wedge \boldsymbol{\omega}_{b}^{a}-\boldsymbol{\omega} \wedge \mathbf{e}^{a} \\
\mathbf{S}_{a} & =\mathbf{d} \mathbf{f}_{a}-\boldsymbol{\omega}^{b}{ }_{a} \wedge \mathbf{f}_{b}+\boldsymbol{\omega} \wedge \mathbf{f}_{a} \\
\boldsymbol{\Omega} & =\mathbf{d} \boldsymbol{\omega}-\mathbf{e}^{a} \wedge \mathbf{f}_{a}
\end{aligned}
$$

where the principal bundle structure allows each curvature to be expanded quadratically in the solder forms, e.g.,

$$
\boldsymbol{\Omega}_{b}^{a}=\frac{1}{2} \Omega_{b c d}^{a} \mathbf{e}^{c} \wedge \mathbf{e}^{d}
$$

Each of the 15 Cartan equations has an integrability condition arising from the Poincaré lemma, $\mathbf{d}^{2} \equiv 0$. For example, the exterior derivative of the torsion is

$$
\mathbf{d} \mathbf{T}^{a}=\mathbf{d}^{2} \mathbf{e}^{a}-\mathbf{d} \mathbf{e}^{b} \wedge \boldsymbol{\omega}_{b}^{a}+\mathbf{e}^{b} \wedge \mathbf{d} \boldsymbol{\omega}_{b}^{a}-\mathbf{d} \boldsymbol{\omega} \wedge \mathbf{e}^{a}+\boldsymbol{\omega} \wedge \mathbf{d} \mathbf{e}^{a}
$$

Using the Poincaré lemma to set $\mathbf{d}^{2} \mathbf{e}^{a} \equiv 0$, and substituting from eqs.(14), (15) and (17), all terms except those linear in curvatures cancel, leaving

$$
\mathbf{d} \mathbf{T}^{a}=-\mathbf{T}^{b} \wedge \boldsymbol{\omega}_{b}^{a}+\boldsymbol{\omega} \wedge \mathbf{T}^{a}+\mathbf{e}^{b} \wedge \boldsymbol{\Omega}_{b}^{a}-\boldsymbol{\Omega} \wedge \mathbf{e}^{a}
$$


Carrying out similar calculations for the remaining Cartan equations, eqs.(14), (16) and (17), we find

$$
\begin{aligned}
0 & =\mathbf{D} \boldsymbol{\Omega}_{b}^{a}+2 \Delta_{d b}^{a c}\left(\boldsymbol{\Omega}_{c} \wedge \mathbf{e}^{d}-\mathbf{f}_{c} \wedge \boldsymbol{\Omega}^{d}\right) \\
0 & =\mathbf{D} \mathbf{T}^{a}-\mathbf{e}^{b} \wedge \boldsymbol{\Omega}_{b}^{a}+\boldsymbol{\Omega} \wedge \mathbf{e}^{a} \\
0 & =\mathbf{D S}_{a}+\boldsymbol{\Omega}^{b} \wedge \mathbf{f}_{b}-\mathbf{f}_{a} \wedge \boldsymbol{\Omega} \\
0 & =\mathbf{D} \boldsymbol{\Omega}+\mathbf{T}^{a} \wedge \mathbf{f}_{a}-\mathbf{e}^{a} \wedge \mathbf{S}_{a}
\end{aligned}
$$

where $\mathbf{D}$ is the Weyl covariant derivative,

$$
\begin{aligned}
\mathbf{D} \boldsymbol{\Omega}_{b}^{a} & =\mathbf{d} \boldsymbol{\Omega}_{b}^{a}+\boldsymbol{\Omega}_{b}^{c} \wedge \boldsymbol{\omega}_{c}^{a}-\boldsymbol{\Omega}_{c}^{a} \wedge \boldsymbol{\omega}_{b}^{c} \\
\mathbf{D} \mathbf{T}^{a} & =\mathbf{d} \mathbf{T}^{a}+\mathbf{T}^{b} \wedge \boldsymbol{\omega}_{b}^{a}-\boldsymbol{\omega} \wedge \mathbf{T}^{a} \\
\mathbf{D} \mathbf{S}_{a} & =\mathbf{d} \mathbf{S}_{a}-\boldsymbol{\omega}_{a}^{b} \wedge \mathbf{S}_{b}+\mathbf{S}_{a} \wedge \boldsymbol{\omega} \\
\mathbf{D} \boldsymbol{\Omega} & =\mathbf{d} \boldsymbol{\Omega}
\end{aligned}
$$

When one of the curvature 2-forms is zero, the Bianchi identities give algebraic relations. Thus, with vanishing torsion, $\mathbf{T}^{a}=0$, eq.(19) becomes

$$
\begin{aligned}
0 & =-\mathbf{e}^{b} \wedge \boldsymbol{\Omega}_{b}^{a}+\boldsymbol{\Omega} \wedge \mathbf{e}^{a} \\
& =-\frac{1}{2} \Omega_{b c d}^{a} \mathbf{e}^{b} \wedge \mathbf{e}^{c} \wedge \mathbf{e}^{d}+\frac{1}{2} \Omega_{c d} \mathbf{e}^{a} \wedge \mathbf{e}^{c} \wedge \mathbf{e}^{d} \\
& =\frac{1}{2}\left(-\Omega_{b c d}^{a}+\delta_{b}^{a} \Omega_{c d}\right) \mathbf{e}^{b} \wedge \mathbf{e}^{c} \wedge \mathbf{e}^{d}
\end{aligned}
$$

so that

$$
\Omega_{[b c d]}^{a}=\delta_{[b}^{a} \Omega_{c d]}
$$

\section{Appendix B: Homogeneous Weyl invariance of the action}

In the linear $S O(4,2)$ representation, an infinitesmial conformal transformation takes the form

$$
g_{B}^{A}=\delta_{B}^{A}+\Lambda_{B}^{A}
$$

where $A, B=0,1, \ldots 5$. With $a, b=0,1,2,3$, we let $\Lambda_{b}^{a}$ be an infinitesimal local Lorentz transformation, $\Lambda^{a} \equiv \Lambda_{4}^{a}$ a local translation, $\Lambda_{a} \equiv \Lambda_{a}^{4}$ a local special conformal transformation, and $\Lambda \equiv \Lambda_{4}^{4}$ a local dilatation. Antisymmetry of the generators allows us to write the remaining $\Lambda_{B}^{A}$ in terms of these. Then the infinitesimal gauge transformations of the conformal connection forms are given by

$$
\begin{aligned}
\delta \boldsymbol{\omega}_{b}^{a}= & \left(\Lambda_{c}^{a}{ }_{c} \boldsymbol{\omega}_{b}-\boldsymbol{\omega}_{c}^{a} \Lambda_{b}^{c}\right)+\left(\Lambda^{a} \mathbf{f}_{b}-\mathbf{e}^{a} \Lambda_{b}\right) \\
& +\eta^{a c} \eta_{b d}\left(\Lambda_{c} \mathbf{e}^{d}-\mathbf{f}_{c} \Lambda^{d}\right)-\mathbf{d} \Lambda_{b}^{a} \\
\delta \mathbf{e}^{a}= & \Lambda_{c}^{a} \mathbf{e}^{c}+\Lambda^{a} \boldsymbol{\omega}-\boldsymbol{\omega}^{a} \Lambda^{c}-\mathbf{e}^{a} \Lambda-\mathbf{d} \Lambda^{a} \\
\delta \mathbf{f}_{b}= & \Lambda_{c} \boldsymbol{\omega}_{b}^{c}+\Lambda \mathbf{f}_{b}-\mathbf{f}_{c} \Lambda_{b}^{c}-\boldsymbol{\omega} \Lambda_{b}-\mathbf{d} \Lambda_{b} \\
\delta \boldsymbol{\omega}= & \Lambda_{c} \mathbf{e}^{c}-\mathbf{f}_{c} \Lambda^{c}-\mathbf{d} \Lambda
\end{aligned}
$$

The auxiliary gauging breaks the translational symmetry. Without the translations these reduce to

$$
\begin{aligned}
\delta \boldsymbol{\omega}_{b}^{a} & =\Lambda_{c}^{a} \boldsymbol{\omega}_{b}^{c}-\boldsymbol{\omega}_{c}^{a} \Lambda_{b}^{c}-\mathbf{e}^{a} \Lambda_{b}+\eta^{a c} \eta_{b d} \Lambda_{c} \mathbf{e}^{c}-\mathbf{d} \Lambda_{b}^{a} \\
\delta \mathbf{e}^{a} & =\Lambda_{c}^{a} \mathbf{e}^{c}-\mathbf{e}^{a} \Lambda \\
\delta \mathbf{f}_{b} & =\Lambda_{c} \boldsymbol{\omega}_{b}^{c}+\Lambda \mathbf{f}_{b}-\mathbf{f}_{c} \Lambda_{b}^{c}-\boldsymbol{\omega} \Lambda_{b}-\mathbf{d} \Lambda_{b} \\
\delta \boldsymbol{\omega} & =\Lambda_{c} \mathbf{e}^{c}-\mathbf{d} \Lambda
\end{aligned}
$$


showing that the solder form has become tensorial.

For the curvatures, the $\mathcal{I} \mathcal{W}$ transformations are similar,

$$
\begin{aligned}
\delta \boldsymbol{\Omega}_{b}^{a} & =\Lambda_{c}^{a} \boldsymbol{\Omega}_{b}^{c}-\boldsymbol{\Omega}_{c}^{a} \Lambda_{b}^{c}-\mathbf{T}^{a} \Lambda_{b}+\eta^{a c} \eta_{b d} \Lambda_{c} \mathbf{T}^{d} \\
\delta \mathbf{T}^{a} & =\Lambda_{c}^{a} \mathbf{T}^{c}-\mathbf{T}^{a} \Lambda \\
\delta \mathbf{S}_{b} & =\Lambda_{c} \boldsymbol{\Omega}_{b}^{c}+\Lambda \mathbf{S}_{b}-\mathbf{S}_{c} \Lambda_{b}^{c}-\boldsymbol{\Omega} \Lambda_{b} \\
\delta \boldsymbol{\Omega} & =\Lambda_{c} \mathbf{T}^{c}
\end{aligned}
$$

Notice that if we suppress special conformal transformations, $\Lambda_{a}=0$, both the Lorentz curvature and dilatational curvature become separate tensors under the remaining $\mathcal{W}$ transformations so the action eq.(3) is manifestly $\mathcal{W}$-invariant. The translational symmetry has been replaced by general coordinate invariance of the curved manifold [34].

For a full $\mathcal{I W}$ transformation of the action we easily show that

$$
\delta S_{\text {auxiliary }}^{\mathcal{W}}=-4 \alpha \int \Lambda_{b} \mathbf{T}^{a} \wedge^{*} \boldsymbol{\Omega}^{b}{ }_{a}+2 \beta \int \Lambda_{c} \mathbf{T}^{c} \wedge^{*} \boldsymbol{\Omega}
$$

Therefore, $S_{\text {auxiliary }}^{\mathcal{W}}$ is invariant if we perform no special conformal transformations, $\Lambda_{b}=0$, or if the torsion vanishes, $\mathbf{T}^{a}=0$.

\section{Appendix C: The basis structure equation and the connection}

Various relations between the solder form, metric, and connection are readily established from the basis structure equation, eq.(15),

$$
\mathbf{d e}^{a}=\mathbf{e}^{b} \wedge \boldsymbol{\omega}_{b}^{a}+\boldsymbol{\omega} \wedge \mathbf{e}^{a}+\mathbf{T}^{a}
$$

Eq. (15) gives the antisymmetric part of the partial derivative of the solder form, so we must have

$$
\partial_{\nu} e_{\mu}{ }^{a}+e_{\mu}{ }^{b} \omega_{b \nu}^{a}-W_{\nu} e_{\mu}^{a}+\frac{1}{2} T_{\mu \nu}^{a}=\Sigma_{\mu \nu}^{a}
$$

where $\Sigma_{\mu \nu}^{a}$ is symmetric, $\Sigma_{\mu \nu}^{a}=\Sigma_{\nu \mu}^{a}$. Permuting indices and combining to solve for $\Sigma_{\mu \nu}^{a}$ in the usual way leads to

$$
\Sigma_{\mu \alpha \nu}=\frac{1}{2}\left(\partial_{\nu} g_{\alpha \mu}+\partial_{\alpha} g_{\mu \nu}-\partial_{\mu} g_{\nu \alpha}\right)-\left(g_{\alpha \mu} W_{\nu}+g_{\mu \nu} W_{\alpha}-g_{\nu \alpha} W_{\mu}\right)+\frac{1}{2}\left(T_{\alpha \mu \nu}+T_{\nu \mu \alpha}\right)
$$

and substituting back into the derivative of the solder form, we have

$$
D_{\nu} e_{\mu}{ }^{a} \equiv \partial_{\nu} e_{\mu}{ }^{a}+e_{\mu}{ }^{b} \omega_{b \nu}^{a}-e_{\alpha}{ }^{a} \tilde{\Gamma}_{\mu \nu}^{\alpha}-e_{\mu}{ }^{a} W_{\nu}=0
$$

where we define the connection (for a Weyl geometry with torsion) as

$$
\tilde{\Gamma}_{\mu \nu}^{\beta} \equiv \Gamma_{\mu \nu}^{\beta}-\left(\delta_{\mu}^{\beta} W_{\nu}+\delta_{\nu}^{\beta} W_{\mu}-g^{\alpha \beta} g_{\nu \mu} W_{\alpha}\right)+\frac{1}{2}\left(T_{\mu \nu}^{\beta}+T_{\nu \mu}^{\beta}-T_{\mu \nu}^{\beta}\right)
$$

As expected,

$$
\tilde{\Gamma}_{\mu \nu}^{\beta}-\tilde{\Gamma}_{\nu \mu}^{\beta}=-T_{\mu \nu}^{\beta}
$$

Contracting $D_{\nu} e_{\mu}{ }^{a}$ with a second solder form and symmetrizing, we have metric compatibility,

$$
D_{\nu} g_{\alpha \mu}=\partial_{\nu} g_{\alpha \mu}-g_{\alpha \beta} \tilde{\Gamma}_{\mu \nu}^{\beta}-g_{\mu \beta} \tilde{\Gamma}_{\alpha \nu}^{\beta}=0
$$

and standard manipulations (e.g., [35]) show that

$$
\partial_{\alpha}(\sqrt{-g})=\sqrt{-g} \tilde{\Gamma}_{\mu \alpha}^{\mu}
$$




\section{References}

[1] Charles W. Misner, Kip S. Thorne, and John Archibald Wheeler, Gravitation, W. H. Freeman and Company (1973) 431.

[2] Weyl, Hermann, Sitz. Königlich Preußischen Akademie Wiss. (1918) 465; H. Weyl, Ann. d. Physik (4) 59, (1919) 101; H. Weyl, Gött. Nachr. (1921) 99; H. Weyl, Raum, Zeit, Materie, Springer, Berlin, (1919-1923) .

[3] Hermann Weyl, Math. Zeitschr., 2 (1918b) 384.

[4] Rudolf Bach, Mathematische Zeitschrift 9 (1-2) 110.

[5] W. Fenchel, (I), J. London Math. Soc. vol. 15 (1940) 15; C. B. Allendoerfer, Amer. J. Math. vol. 62 (1940) 243.; Carl B. Allendoerfer and André Weil. Trans. Amer. Math. Soc. 53 (1943) 101.

[6] C. M. Bender, P. D. Mannheim, Phys. Rev. Lett. 100, 110402 (2008).

[7] A. V. Smilga, SIGMA 5, 017 (2009).

[8] P. D. Mannheim, Prog. Part. Nucl. Phys. 56, (2006) 340.

[9] Éanna É. Flanagan, Phys.Rev. D74 (2006) 023002l.

[10] Joseph Sultana, Demosthenes Kazanas, and Jackson Levi Said, Phys Rev D 86, (2012) 084008.

[11] J. Isberg, U. Lindström and B. Sundborg, Phys. Lett. B 293 (1992) 321; J. Isberg, U. Lindström B. Sundborg and G. Theodoridis, Nucl. Phys. B 411 (1994) 122; H. Gustafsson, U. Lindström, P. Saltsidis, B. Sundborg and R.v. Unge, Nucl. Phys. B 440 (1995) 495.

[12] Brinkmann, H. W., Math. Ann. 91 (1924) 269.

[13] P. Szekeres, Proc Roy Soc London. Series A, Mathematical and Physical Sciences, 274, 1357 (1963) 206.

[14] C. Kozameh, E.T. Newman and K.P. Tod, Gen.Rel.Grav. 17 (1985) 343; M. Listing, Annals of Global Analysis and Geometry, 20, (2001) 183; Carlos Kozameh, Ezra T. Newman, Pawel Nurowski, Class.Quant.Grav. 20 (2003) 3029; A.R. Grover and P. Nurowski, math/0405304[math-dg].

[15] Hans-Jürgen Schmidt, Annalen der Physik, Annalen Phys. 41 (1984) 435.

[16] Nurowski P, Plebanski J F, Class. Q. Grav. 18, (2001) 341.

[17] Hai-Shan Liu, H. Lü, C.N. Pope and J. F. Vázquez-Poritz, arXiv:1303.5781,

[18] Jorge Crispim Romao, Alan Ferber, and Peter G. O. Freund, Nucl.Phys. B126 (1977) 429.

[19] M. Kaku, P. K. Townsend and P. van Nieuwenhuizen, Phys. Lett. B 69 (1977) 304.

[20] M. Kaku, P.K. Townsend and P. van Nieuwenhuizen, Phys. Rev. D17 (1978) 3179.

[21] Jorge Crispim-Romao, Nuc.Phys.B145 (1978) 535.

[22] Conformal supergravity E.S. Fradkin, A.A. Tseytlin, Physics Reports Volume 119, Issues 4-5, March 1985, Pages 233-362

[23] James T. Wheeler, Phys. Rev. D 446 (1991) 1769.

[24] E. A. Ivanov and J. Niederle, Phys.Rev.D, Vol. 25, 4, (1982) 976. 
[25] E. A. Ivanov and J. Niederle, Phys.Rev.D, 25, 4 (1982) 988.

[26] Y. Ne'eman and T. Regge, Phys.Lett.B 74 1-2 (1978) 54.

[27] S. Kobayashi and K. Nomizu, Foundations of Differential Geometry, John Wiley and Sons (1963).

[28] Wheeler, James T., J. Math. Phys. 39 (1998) 299.

[29] André Wehner and James T. Wheeler, Nuc. Phys. B 557 (1999) 380.

[30] C. Lanczos, Ann. Math., 394 (1938) 842.

[31] David Lovelock, Proc. Camb. Phil. Soc., 68 (1970) 345.

[32] Juan Trujillo and James T. Wheeler, in preparation.

[33] Levi-Civita, T., Rom. Acc. L. Rend. (5) 272 (1918) 183.

[34] Kibble, T. W. B., J. Math. Phys. 2 (1961) 212-221.

[35] S. Weinberg, Gravitation and Cosmology, John Wiley and Sons, (1972). 